\title{
MONITORAMENTO EM TEMPO REAL DA UMIDADE DO PÓ DURANTE A SECAGEM DE PASTAS EM LEITO DE JORRO UTILIZANDO UM SENSOR VIRTUAL HÍBRIDO NEURAL
}

\author{
G. N. A. VIEIRA ${ }^{1,2^{*}}$, F. B. FREIRE ${ }^{2}$, J. T. FREIRE ${ }^{2}$, \\ ${ }^{1}$ Universidade Federal de Alfenas, Instituto de Química \\ ${ }^{2}$ Universidade Federal de São Carlos, Departamento de Engenharia Química \\ e-mail: gustavo.vieira@unifal-mg.edu.br
}

\begin{abstract}
RESUMO
Do ponto de vista do controle do processo, seria mais interessante controlar diretamente a umidade do produto seco em processos de secagem. Entretanto, trata-se de um controle de concentração, que comumente depende de procedimentos lentos ou de alto custo. Uma forma de reduzir estes custos é utilizar outros sensores, cuja resposta seja mais rápida, que tenham menor custo e que possam fornecer informações necessárias a um modelo físico-matemático que represente o processo. Neste sentido, objetivou-se neste trabalho o desenvolvimento e aplicação de um sensor virtual para a medição online da umidade do pó produzido em um leito de jorro utilizado para a secagem de pastas. Para o cumprimento deste objetivo, primeiramente foram feitos experimentos de secagem de leite integral, para que se determinasse o comportamento dinâmico do processo de secagem analisado. Posteriormente, foi satisfatoriamente ajustado um modelo híbrido neural para a secagem de leite bovino integral, para ser utilizado como parte do sensor virtual. O sensor foi capaz de estimar a umidade do pó produzido pelo secador, mesmo quando este foi submetido a diferentes perturbações em malha aberta.
\end{abstract}

\section{INTRODUÇÃO}

Em secadores, uma das variáveis de processo mais importantes é a umidade do produto na saída do secador, pois ela é um fator que exerce grande influência sobre as etapas posteriores de processamento, sobretudo àquelas relacionadas com sua qualidade. Por exemplo, produtos agroindustriais que apresentam alta umidade podem sofrer contaminação microbiológica, causando grandes perdas. Caso a umidade seja mais baixa que a especificada, o produto apresentará não só custos de produção maiores (já que mais recursos energéticos foram gastos), mas também poderá apresentar propriedades indesejadas, tais como dano térmico, propriedades relacionadas à reidratação (CHEGINI et al., 2008), etc.

Apesar da grande importância dessa variável, sua medição para fins de controle não é facilmente aplicável a qualquer produto. Alguns dos exemplos de medidores de umidade online são aqueles baseados em algumas propriedades físicas da água, dentre as quais se destaca sua capacidade de absorver radiação eletromagnética, principalmente na faixa de frequências do infravermelho e das micro-ondas (CARRBRION, 1986). Gradualmente, estes medidores estão sendo utilizados na indústria química (BLOCK; LIPTÁK; SHINSKEY, 2006; BRODGESELL; LIPTÁK, 2006). Porém, infelizmente, as técnicas de medição 
online de umidade por radiação eletromagnética não são de simples aplicação a produtos secos particulados (sementes, pós etc.), pois os espaços vazios entre as partículas tornam a medição ruidosa, devido à reflexão difusa da radiação. Além disso, tais equipamentos são normalmente de alto custo.

Outra dificuldade da medição da umidade de sólidos visando ao controle de processos de secagem é que os métodos convencionais e de baixo custo para a medição da umidade são lentos, isto é, não apresentam a frequência necessária para a implementação prática de ações de controle. De fato, um dos métodos mais comuns é o gravimétrico em estufa, cujo tempo de análise é muito grande (da ordem de horas). Consequentemente, o controle da umidade por amostragem é impraticável, por haver grande perda de informação enquanto a medida é feita. Em outras palavras, enquanto uma análise é realizada, o processo opera em malha aberta, sem correção sobre os desvios da variável controlada.

Mesmo com este contexto desfavorável, o controle da umidade de sólidos particulados em secadores pode encontrar no desenvolvimento de técnicas computacionais uma solução para esse impasse: o uso de modelos físico-matemáticos com resolução em tempo real permite inferir valores de variáveis de difícil medição a partir de outras mais facilmente medidas. Com isso, é possível desenvolver um sensor virtual, utilizando outros dados cuja frequência de aquisição de dados seja suficientemente alta para que, no caso de secadores, a umidade seja efetivamente uma variável controlada em processos de secagem.

Sendo assim, este estudo visa à incorporação de um modelo matemático à aquisição de dados em um leito de jorro aplicado à secagem de pastas, visando ao desenvolvimento de um sensor virtual de umidade do pó, que poderá ser utilizado em estratégias de controle inferencial.

\section{MATERIAIS E MÉTODOS}

\subsection{Equipamento}

A Figura 1 mostra um esquema do secador de leito de jorro utilizado neste trabalho.

O secador foi alimentado com ar por um soprador (1) de $7 \mathrm{hp}$. A vazão foi medida por uma placa de orifício (2), na qual se mediu a queda de pressão, utilizando um transmissor de pressão (3). Outro medidor semelhante foi utilizado para medir a queda de pressão no leito. $\mathrm{O}$ ar foi aquecido por resistores elétricos (4) e foi alimentado ao leito de jorro (5). A fase sólida do leito consistia em partículas de polietileno, com diâmetro médio de $4,38 \mathrm{~mm}$ e densidade $940 \mathrm{~kg} / \mathrm{m}^{3}$.

Figura 1 - Diagrama esquemático do leito de jorro utilizado neste trabalho.

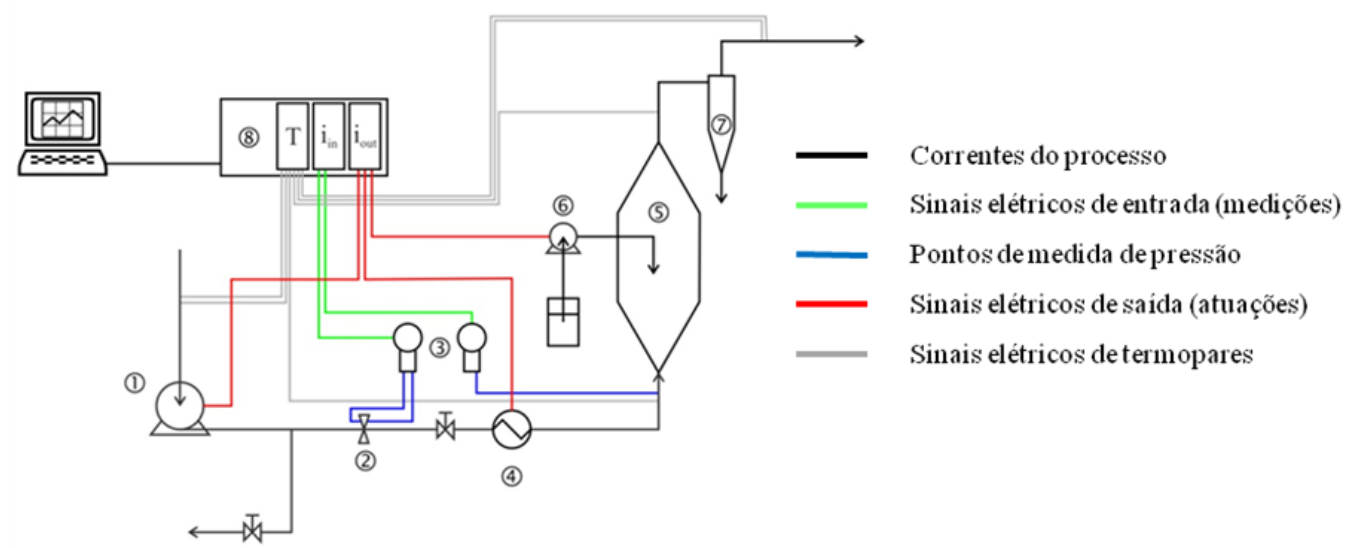

Fonte: Autor (2015). 
As dimensões do leito de jorro são mostradas na Tabela 1.

Tabela 1 - Dimensões características do leito de jorro utilizado neste trabalho.

\begin{tabular}{lr}
\multicolumn{1}{c}{ Dimensão } & Valor \\
\hline $\boldsymbol{H}(\mathbf{m})$ & 1,00 \\
$\boldsymbol{D}_{\boldsymbol{c}}(\mathbf{m})$ & 0,30 \\
$\boldsymbol{d}_{\boldsymbol{i}}(\mathbf{m})$ & 0,03 \\
$\boldsymbol{\theta}\left({ }^{\circ}\right)$ & 60 \\
$\boldsymbol{H}(\mathbf{m})$ & 1,00 \\
\hline
\end{tabular}

Fonte: Autor (2015).

Quando necessária, a pasta foi alimentada no secador utilizando-se uma bomba dosadora solenoide (6), que alimentava a pasta a partir de um reservatório de pasta até um atomizador de duplo fluido. Ar comprimido com pressão de $1,0 \mathrm{kgf} / \mathrm{cm}^{2}$ foi utilizado para atomizar a pasta no secador. A pasta seca foi recolhida do sistema utilizando um ciclone (7).

A pasta utilizada neste trabalho foi o leite bovino integral ultrapasteurizado, adquirido em mercado local. Escolheu-se esta pasta devido à grande importância econômica do produto final (leite em pó) e da facilidade de aquisição da pasta.

Todos os dados experimentais eram obtidos uma placa de aquisição de dados (8). Os dados obtidos por esta placa eram monitorados em uma interface desenvolvida no software LabVIEW 2011 SP1. Nesta interface, foi possível medir as temperaturas e umidades do ar, utilizando termopares tipo $\mathrm{T}$ (ilustrados por linhas cinza na Figura 1) e as quedas de pressão medida pelos transmissores de pressão (linhas azuis). Além disso, a interface permite estabelecer sinais de corrente elétrica (linhas vermelhas) aos atuadores, que não foram ilustrados na Figura 1 para fins de clareza: para o estabelecimento da vazão de ar, utilizou-se um inversor de frequência; para o estabelecimento da potência dissipada nos resistores elétricos, utilizou-se um módulo de potência tiristorizado; e para o estabelecimento da vazão de pasta, os sinais recebidos pela bomba dosadora solenoide estavam relacionados a diferentes frequências de batimento do diafragma da bomba.

\subsection{Procedimento experimental}

Primeiramente, realizou-se a caracterização fluidodinâmica do leito de jorro pelo procedimento clássico descrito por Epstein e Grace (2011), para a obtenção da velocidade mínima de jorro. Desta forma, estabeleceu-se a vazão de ar durante os experimentos de secagem como sendo equivalente a $115 \%$ da vazão mínima de jorro.

\subsubsection{Verificação de um modelo híbrido} neural

O modelo híbrido neural utilizado neste trabalho foi o proposto por Freire et al. (2012). O procedimento experimental acima descrito foi realizado previamente pelos autores, que verificaram que o modelo é representativo do processo.

De forma a verificar se o modelo é capaz de incorporar novos dados, o procedimento foi repetido para outras condições experimentais. Primeiramente, a operação do secador foi iniciada ao se estabelecer a vazão de ar na entrada do secador em $115 \%$ da vazão mínima de jorro $\left(2,30 \mathrm{~m}^{3} / \mathrm{min}\right)$ e a temperatura na entrada do secador como sendo $90^{\circ} \mathrm{C}$. Quando o regime permanente foi atingido, iniciou-se a alimentação da pasta inicialmente à vazão de $20 \mathrm{~mL} / \mathrm{min}$. Foram obtidos então os dados relacionados ao ar na saída do secador (temperatura e umidade) a cada 20 segundos, com o uso da interface desenvolvida em LabVIEW 2011 SP1. Cada ponto experimental deste trabalho obtido por esta interface corresponde à média de 500 pontos obtidos a uma frequência de $1,0 \mathrm{kHz}$. 
Paralelamente a estas medidas, coletaram-se amostras do pó recolhido pelo ciclone a cada $10 \mathrm{~min}$, submetendo-as à análise de umidade pelo método proposto pelo Instituto Adolfo Lutz (2008) para amostras de leite e derivados. Este método consiste na secagem da amostra em estufa a $93^{\circ} \mathrm{C}$ até massa constante. Preferiu-se utilizar este método devido ao fato de que o método gravimétrico em estufa "convencional" $\left(105^{\circ} \mathrm{C}\right.$ por $\left.24 \mathrm{~h}\right)$ causou forte escurecimento na amostra, que pode afetar negativamente $o$ resultado da análise.

Após cada experimento, os inertes foram retirados e lavados com água corrente em abundância, de modo a retirar o pó sobre as partículas. Posteriormente, um novo experimento foi realizado com uma vazão de pasta aumentada em $10 \mathrm{~mL} / \mathrm{min}$, repetindo-se todo o procedimento até que fossem notadas instabilidades no secador, devido ao acúmulo de pasta.

O modelo híbrido neural é constituído das seguintes equações:

$$
\begin{aligned}
& F=F_{i}-k \\
& W=W_{i}+k \\
& \frac{d y}{d t}=\frac{1}{m_{g}}\left(W_{i} y_{i}-W y+k\right) \\
& x=\frac{F_{i} x_{i}-k}{F} \\
& \frac{d T}{d t}=\frac{W c_{g i} T_{i}+F_{i} c_{p i} T_{\infty}-W c_{g} T-F c_{p} T-k \lambda-h A_{l}\left(T-T_{\infty}\right)}{m_{g} c_{g}+m_{s} c_{s}+m_{j} c_{j}}
\end{aligned}
$$

As principais hipóteses do modelo são: (a) o leito de jorro se comporta como um tanque de mistura perfeita; (b) o ar úmido se comporta como uma mistura de gases ideais (ar seco com vapor d'água, para o cálculo dos calores específicos sensíveis no balanço de energia); (c) a difusão da água sobre a película de pasta que recobre as partículas é desprezível. Mais detalhes sobre este modelo podem ser encontrados em Almeida (2009), Freire et al. (2012) e Nascimento (2013).

O modelo foi ajustado aos dados experimentais, com base no treinamento da rede neural. Uma parcela dos dados obtidos por Nascimento (2013) foi utilizada conjuntamente com os dados deste trabalho para o treinamento da rede neural.

O termo de acoplamento de fases $k$ foi estimado por uma rede neural, descrevendo a dinâmica da secagem em função da natureza da pasta, do tempo de injeção de pasta $(t)$, da temperatura do ar na entrada do secador $\left(T_{i}\right)$ e da vazão de pasta alimentada no sistema $\left(F_{i}\right)$. A arquitetura da rede neural utilizada neste trabalho é esquematizada na Figura 2.

Figura 2 - Esquema da rede neural.

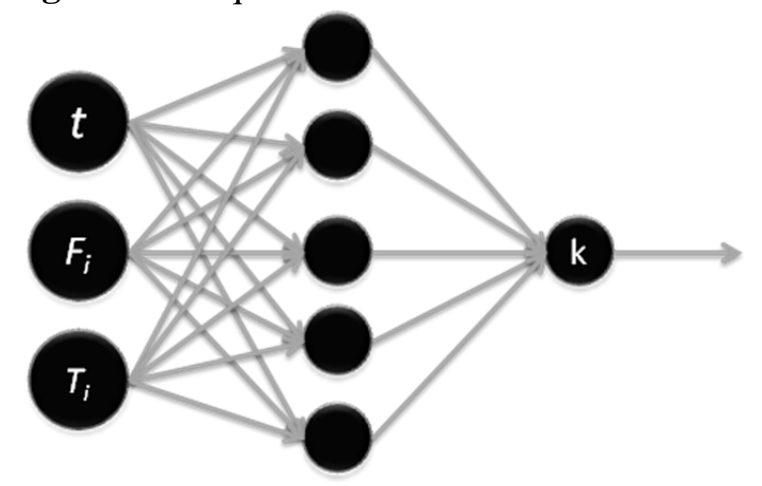

Fonte: Autor (2015).

A rede neural consiste em três neurônios na camada de entrada, relacionados ao tempo de injeção de pasta, temperatura do ar na entrada do secador e da vazão de pasta. Utilizaram-se cinco neurônios na camada intermediária e um na camada de saída, que fornece o termo de acoplamento de fases.

Para o treinamento da rede neural, foi utilizada a função "nftool" do software MATLAB R2007b. Esta função é responsável pela minimização do erro quadrático médio em relação aos targets (termo de acoplamento de fases obtido com base no modelo proposto por Almeida (2009), ajustado previamente). O 
algoritmo de minimização padrão desta função no software é o de LevenbergMarquardt. Os dados foram divididos em três partes: $60 \%$ deles foram utilizados na etapa de treinamento, $20 \%$ na parte de validação e os $20 \%$ restantes na etapa de teste.

Mais detalhes sobre a estrutura da rede neural, bem como o procedimento utilizado para seu treinamento podem ser encontrados em Vieira (2015).

Obtida a rede neural que descreve o termo de acoplamento de fases, o modelo híbrido neural foi resolvido numericamente utilizando o mesmo software pelo método de Runge-Kutta (função "ode45” do software), utilizando como condições iniciais os dados experimentais no início dos experimentos acima descritos.

2.2.2 Implementação do sensor virtual na interface do usuário

Após a verificação do modelo híbrido neural, ele foi incorporado à interface do usuário em LabVIEW 2011 SP1, com cálculos auxiliares (uso da rede neural) feitos simultaneamente pelo mesmo software utilizado para o treinamento da rede neural (MATLAB R2007b). Com base no modelo híbrido neural e nos dados obtidos pela placa de aquisição de dados, a interface forneceu valores da umidade do pó estimados pelo modelo.

De forma a analisar se o modelo foi capaz de fornecer boas estimativas da umidade do pó, realizaram-se experimentos adicionais. Após o estabelecimento do regime permanente com o sistema inicialmente alimentado por ar a $90^{\circ} \mathrm{C}$, com vazão de ar $115 \%$ da vazão mínima de jorro e $20 \mathrm{~mL} / \mathrm{min}$ de pasta, as variáveis manipuladas do processo foram variadas segundo o esquema mostrado na Figura 3.

Portanto, realizaram-se perturbações na vazão de pasta e na temperatura do ar na entrada do secador sobre o sistema em malha aberta. Durante o tempo em que o sistema foi perturbado, foram feitas as mesmas medições das variáveis do processo descritas anteriormente para a verificação do modelo híbrido neural. A comparação entre o valor da umidade do pó estimada pelo sensor virtual e o obtido pelo método gravimétrico (medida conforme o mesmo procedimento descrito anteriormente) permitiu a verificação da robustez e da precisão do sensor virtual.

Figura 3 - Perturbações a que o secador foi submetido durante os experimentos de monitoramento.

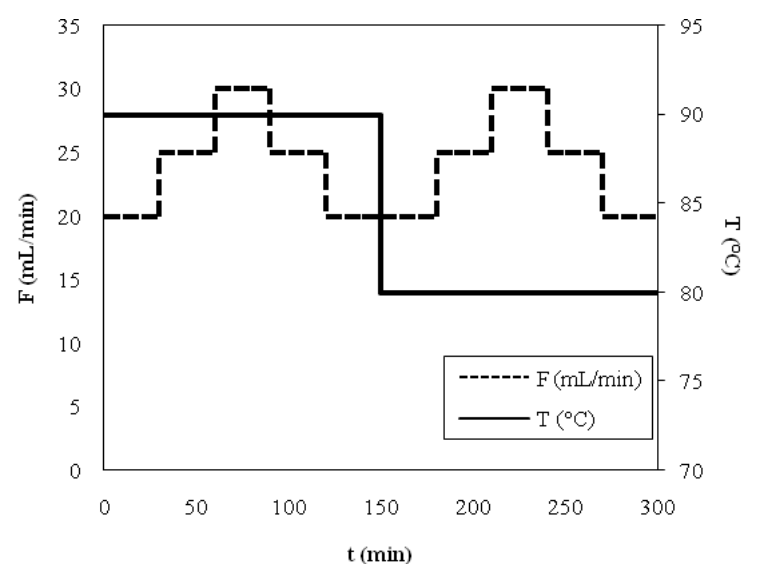

Fonte: Autor (2015).

\section{RESULTADOS E DISCUSSÃO}

\subsection{Verificação do modelo híbrido neural}

A Figura 4 mostra o termo de acoplamento de fases em função do tempo para os experimentos de verificação do modelo. Os dados a $80^{\circ} \mathrm{C}$ são os incorporados a este trabalho (NASCIMENTO, 2013) e os dados a $90^{\circ} \mathrm{C}$ são os referentes ao procedimento acima descrito.

A rede neural foi capaz de calcular com precisão os valores do termo de acoplamento de fases. O coeficiente de correlação $(r)$ nas etapas de treinamento, validação e teste da rede neural foram iguais a 0,9999, 0,9985 e 0,9999 , respectivamente. Isso mostra que houve uma boa correlação entre o modelo e os dados experimentais. 
Figura 4 - Termo de acoplamento de fases em função do tempo para os experimentos de secagem de pasta real em malha aberta a (a) $80^{\circ} \mathrm{C}$ (b) $90^{\circ} \mathrm{C}$.

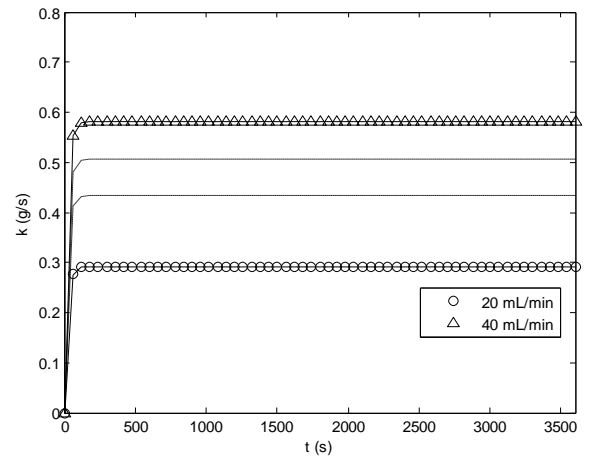

(a)

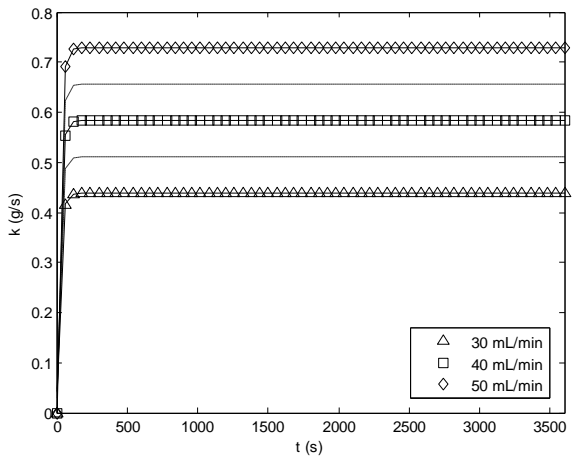

(b)

Fonte: Autor (2015).

Ao se utilizar a rede neural e a equação (4), pôde-se obter então a umidade do pó em função do tempo. A Tabela 2 mostra os dados resultantes da simulação numérica após o estabelecimento do regime permanente, em comparação aos dados experimentais.

Tabela 2 - Comparação entre valores preditos e experimentais das umidades do pó em regime permanente.

\begin{tabular}{ccccc}
\hline $\begin{array}{c}T_{i} \\
\left({ }^{\circ} \mathrm{C}\right)\end{array}$ & $\begin{array}{c}F_{i} \\
(\mathrm{~mL} / \mathrm{min})\end{array}$ & $\begin{array}{c}x \text { (b.u.) } \\
\text { modelo }\end{array}$ & $\begin{array}{c}x \text { (b.u. }) \\
\text { experimental }\end{array}$ & $\begin{array}{c}\text { Erro } \\
(\%)\end{array}$ \\
\hline \multirow{2}{*}{80} & 20 & 0,0625 & 0,0614 & 2,0 \\
& 40 & 0,0832 & 0,0871 & 4,4 \\
\hline \multirow{3}{*}{90} & 30 & 0,0433 & 0,0407 & 6,5 \\
& 40 & 0,0468 & 0,0548 & $-14,3$ \\
& 50 & 0,0628 & 0,0566 & 11,0 \\
\hline
\end{tabular}

Fonte: Autor (2015).

$\mathrm{O}$ erro foi menor que $10 \%$ na maioria dos casos, à exceção de dois experimentos. Ainda assim, o erro pode ser considerado aceitável para predizer a umidade do pó e, portanto, o modelo híbrido neural é um potencial sensor virtual da umidade do pó para fins de controle. Além disso, provou-se que a rede neural foi capaz de incorporar novas condições testadas, visto que o banco de dados obtidos por Nascimento (2013) foi expandido quanto à temperatura do ar na entrada do secador $\left(90^{\circ} \mathrm{C}\right)$.

\subsection{Implementação do sensor virtual na interface do usuário}

A Figura 5 mostra os resultados a partir do experimento de monitoramento da umidade do pó em malha aberta, a partir da implementação do sensor virtual à interface do usuário.

Nos instantes iniciais (até cerca de 150 minutos), o erro na estimativa da umidade dada pelo sensor virtual em relação à medição pelo método gravimétrico foi relativamente pequeno - próximo aos mostrados na Tabela 2 -, podendo ser considerado uma boa estimativa em linha da umidade.

Por outro lado, para tempos longos de secagem (a partir de 150 minutos, tempo correspondente à redução da temperatura), houve um erro consideravelmente maior na estimativa - em alguns casos, aproximadamente iguais a $150 \%$. Apesar desta superestimação da umidade pelo sensor virtual, a tendência de aumento ou diminuição prevista pelo sensor virtual foi de fato observada nas medições fora de linha. 
Figura 5 - Temperatura na entrada do secador e umidade do pó na saída do secador obtida experimentalmente e estimada pelo sensor virtual em função do tempo, durante o experimento de perturbação em malha aberta.

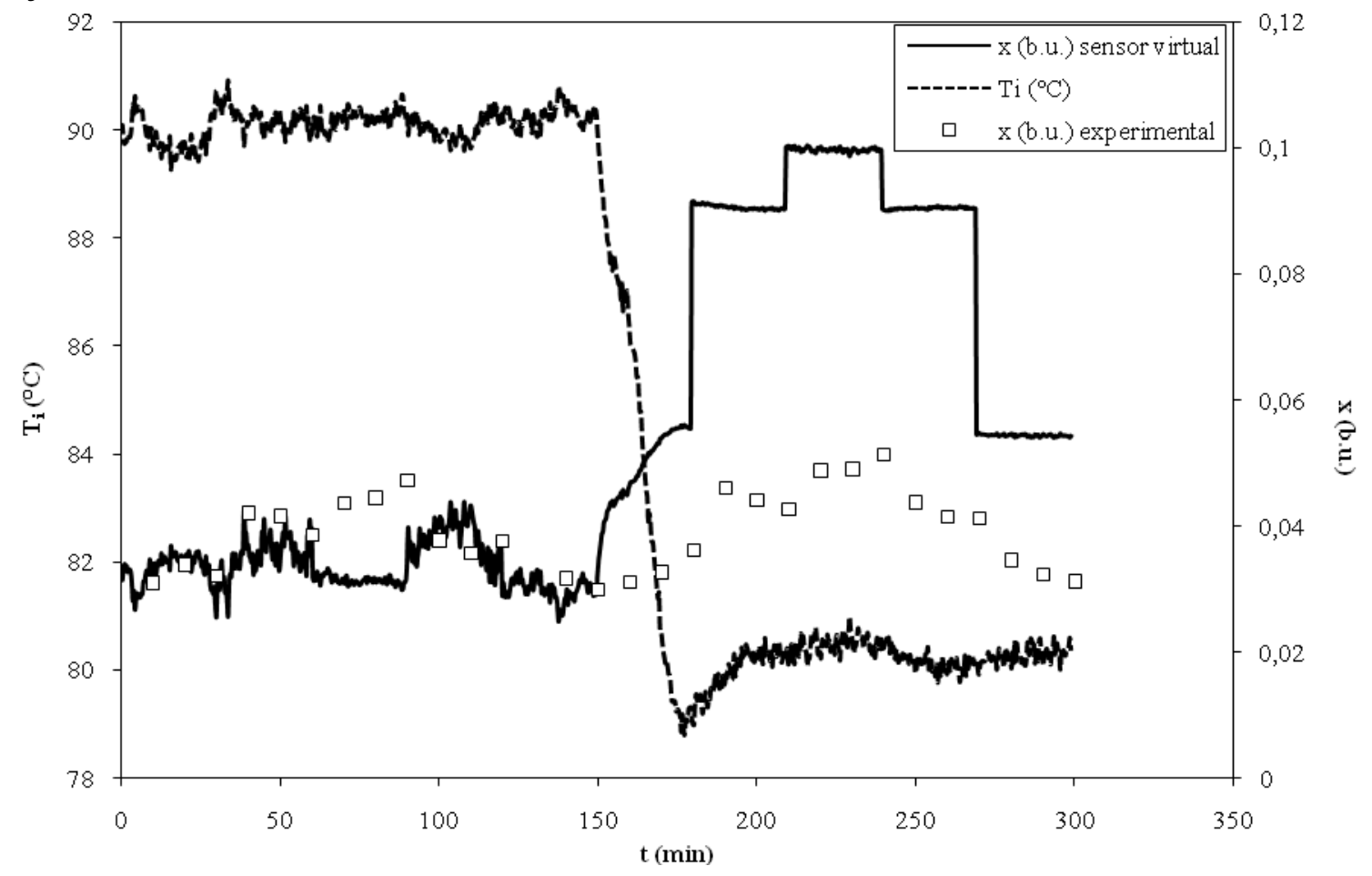

Fonte: Autor (2015).

Este aumento do desvio pode ser explicado se for considerado que, para tempos longos, o pó que recobre as partículas apresenta um "tempo de residência" maior. Desta forma, valores menores de umidade podem ser obtidos. Este pó mais seco foi removido somente após a redução da vazão de pasta para $20 \mathrm{~mL} / \mathrm{min}$. Como o tempo de operação do secador foi relativamente longo neste caso, a remoção deste pó mais seco só foi observada posteriormente. Trata-se de um fenômeno que não foi previsto pelo modelo híbrido neural, portanto, deve ser analisado com mais detalhes para melhoria da precisão do sensor virtual. Mesmo assim, as estimativas fornecidas pelo sensor podem ser consideradas adequadas.

\section{CONCLUSÕES}

A partir do exposto neste trabalho, conclui-se que o modelo híbrido neural previamente proposto foi capaz de incorporar novas condições experimentais e continuar representando bem o processo de secagem de pastas em leito de jorro.

Quando utilizado simultaneamente à aquisição de dados do secador, o modelo híbrido neural se tornou então uma das partes de um sensor virtual, que se provou robusto e preciso nas condições experimentais analisadas. A única exceção encontrada neste trabalho para a boa precisão do sensor virtual ocorreu quando o tempo de secagem foi muito longo. Em todo caso, a ferramenta desenvolvida neste trabalho pode ser utilizada como um procedimento de estimativa em linha da umidade de pós produzidos em secadores de leito de jorro, podendo ser utilizada em estratégias de controle.

Neste trabalho, realizou-se o monitoramento em malha aberta, isto é, sem a atuação de controladores sobre o processo, para que a variável controlada (umidade do 
pó) atinja um valor estabelecido (set point). Devido à relativa robustez e precisão do sensor virtual desenvolvido neste trabalho, poderá ser possível incorporá-lo a uma estratégia de controle inferencial, de modo que a umidade do pó se torne de fato uma variável controlada (com base em estimativas fornecidas pelo sensor virtual).

De fato, já foram realizadas simulações numéricas em malha fechada do controle da umidade do pó produzido pela secagem em leito de jorro, com base neste modelo híbrido neural, utilizando como variável manipulada a temperatura na entrada do aquecedor (VIEIRA et al., 2014). As simulações mostraram uma boa estabilidade do sistema em malha fechada, quando o sistema foi perturbado no set point de umidade (controle servo), na umidade e na temperatura ambiente (controle regulatório). Desta forma, trabalhos futuros podem incluir a implementação do controlador sintonizado no secador real, de modo que a umidade do pó seria controlada em linha, com base na inferência fornecida pelo sensor virtual híbrido neural, sem o uso de sensores reais de umidade, de alto custo.

\section{NOMENCLATURA}

$A_{l} \quad$ Área lateral total do secador

$c \quad$ Calor específico sensível

$D_{c} \quad$ Diâmetro da coluna cilíndrica

$d_{i} \quad$ Diâmetro da entrada do ar

$F \quad$ Vazão de pasta ou produto

$H \quad$ Altura da coluna cilíndrica

$h \quad$ Coeficiente de transferência convectiva de calor parede-ambiente

$k \quad$ Termo de acoplamento de fases

$m$ Massa

$T$ Temperatura [na saída do secador]

$t$ Tempo

$W \quad$ Vazão de ar

$x \quad$ Fração mássica de água na pasta ou produto

\section{Letras gregas}

$\lambda$ Entalpia de vaporização da água

$\theta$ Ângulo da base cônica

\section{Subscritos}

$\infty \quad$ Condições ambientais

$g \quad$ Propriedade da fase gasosa

$i \quad$ Entrada do secador

$j \quad$ Propriedade da parede do leito

$p \quad$ Relativo à pasta ou ao pó

$s \quad$ Propriedade das partículas (fase inerte)

\section{REFERÊNCIAS}

ALMEIDA, A. R. F. Análise da secagem de pastas em leito de jorro. São Carlos: UFSCar, 2009. 165 p.

BLOCK, B.; LIPTÁK, B. G.; SHINSKEY, F. G. Dryer controls. In: Instrument Engineers' Handbook: Process Control and Optimization. Boca Raton: CRC Press, 2006, p. 1904-1913.

BRODGESELL, A.; LIPTÁK, B. G. Moisture in solids. In: Instrument Engineers' Handbook: Process Measurement and Analysis. Boca Raton: CRC Press, 2006, p. 1450-1456.

CARR-BRION, K. Moisture Sensors in Process Control. London: Elsevier Science, 1986.

CHEGINI, G. R.; KHAZAEI, J.; GHOBADIAN, B.; GOUZARDI, A. M. Prediction of process and product parameters in orange juice spray dryer using artificial neural networks. Journal of Food Engineering, v. 84, p. 534-543.

EPSTEIN, N.; GRACE, J. R. (Eds.). Spout and Spout-fluid Beds. New York: Cambridge University Press, 2011. 
FREIRE, J. T.; FREIRE, F. B; FERREIRA, M. C.; NASCIMENTO, B. S. A Hybrid Lumped Parameter/Neural Network Model for Spouted Bed Drying of Pastes with Inert Particles. Drying Technology, v. 30, n. 1112, p. 1342-1353, 2012.

INSTITUTO ADOLFO LUTZ. Leites e derivados. In: ZENEBON, O.; PASCUET, N. S.; TIGLEA, P. Métodos físico-químicos para análise de alimentos. 4. ed. São Paulo: Instituto Adolfo Lutz, 2008. Cap. XXVII, p. 823-881.

NASCIMENTO, B. S. Análise do efeito da composição química na secagem de pasta em leito de jorro. São Carlos: UFSCar, 2013. $112 \mathrm{p}$.

VIEIRA, G. N. A. Monitoramento em tempo real e simulação de controle da umidade de pós produzidos pela secagem de pastas em leito de jorro. São Carlos: UFSCar, 2015, $111 \mathrm{p}$.

VIEIRA, G. N. A.; NASCIMENTO, B. S.; CORRÊA, R. G.; FREIRE, F. B.; FREIRE, J. T. Simulation of a neural network-based inferential controller of moisture content of milk powder produced in a spouted bed dryer. Proceedings of the $19^{\text {th }}$ International Drying Symposium. Lyon: EDP Sciences, 2014.

\section{AGRADECIMENTOS}

Os autores agradecem o apoio financeiro da Fundação de Amparo à Pesquisa do Estado de São Paulo (FAPESP) para a realização desta pesquisa (processo 2011/21413-6). 